\title{
A Novel Enrichment Culture Highlights Core Features of Microbial Networks Contributing to Autotrophic Fe(II) Oxidation Coupled to Nitrate Reduction
}

\author{
Yu-Ming Huang a,b Daniel Strauba, ${ }^{a}$ Andreas Kappler ${ }^{b, d}$ Nicole Smith $^{a}$ \\ Nia Blackwella Sara Kleindienst ${ }^{a}$ \\ aMicrobial Ecology, Center for Applied Geoscience, University of Tuebingen, Tuebingen, Germany; \\ ${ }^{\mathrm{b}}$ Geomicrobiology, Center for Applied Geoscience, University of Tuebingen, Tuebingen, Germany; \\ 'Quantitative Biology Center (QBiC), University of Tuebingen, Tuebingen, Germany; \\ dCluster of Excellence, EXC 2124, "Controlling Microbes to Fight Infections," University of Tübingen, Tübingen, \\ Germany
}

\section{Keywords \\ Fe(II) oxidation · Denitrification · Gallionellaceae · \\ Noviherbaspirillum $\cdot$ Meta'omics}

\begin{abstract}
Fe(II) oxidation coupled to nitrate reduction (NRFO) has been described for many environments. Yet very few autotrophic microorganisms catalysing NRFO have been cultivated and their diversity, as well as their mechanisms for NRFO in situ remain unclear. A novel autotrophic NRFO enrichment culture, named culture BP, was obtained from freshwater sediment. After more than 20 transfers, culture BP oxidized $8.22 \mathrm{mM}$ of Fe(II) and reduced $2.42 \mathrm{mM}$ of nitrate within 6.5 days under autotrophic conditions. We applied metagenomic, metatranscriptomic, and metaproteomic analyses to culture BP to identify the microorganisms involved in autotrophic NRFO and to unravel their metabolism. Overall, twelve metagenome-assembled genomes (MAGs) were constructed, including a dominant Gallionellaceae sp. MAG ( $\geq 71 \%$ relative abundance). Genes and transcripts associated with potential Fe(II) oxidizers in culture BP, identified as a Gallionellaceae sp., Noviherbaspirillum sp., and Thiobacillus sp., were likely involved in metal oxidation
\end{abstract}

karger@karger.com www.karger.com/mip

Karger $\stackrel{\text { ' }}{5}$
(C) 2021 The Author(s)

Published by S. Karger AG, Basel

This is an Open Access article licensed under the Creative Commons Attribution-NonCommercial-4.0 International License (CC BY-NC) (http://www.karger.com/Services/OpenAccessLicense), applicable to the online version of the article only. Usage and distribution for commercial purposes requires written permission. (e.g., cyc2, mtoA), denitrification (e.g., nirK/S, nor $B C$ ), carbon fixation (e.g., $r b c L$ ), and oxidative phosphorylation. The putative $\mathrm{Fe}(\mathrm{II})$-oxidizing protein $\mathrm{Cyc} 2$ was detected for the Gallionellaceae sp. Overall, a complex network of microbial interactions among several Fe(II) oxidizers and denitrifiers was deciphered in culture BP that might resemble NRFO mechanisms in situ. Furthermore, 16S rRNA gene amplicon sequencing from environmental samples revealed 36 distinct Gallionellaceae taxa, including the key player of NRFO from culture BP (approx. 0.13\% relative abundance in situ). Since several of these in situ-detected Gallionellaceae taxa were closely related to the key player in culture BP, this suggests that the diversity of organisms contributing to NRFO might be higher than currently known.

(C) 2021 The Author(s).

Published by S. Karger AG, Basel

\section{Introduction}

Oxidation of ferrous iron [Fe(II)] and reduction of ferric iron $[\mathrm{Fe}(\mathrm{III})]$ contribute to biogeochemical carbon, nitrogen, oxygen and sulphur cycles and can be mediated by specific microorganisms. There are three physiological types of $\mathrm{Fe}(\mathrm{II})$-oxidizing bacteria (FeOB), namely, micro- 
aerophiles, photoferrotrophs, and nitrate-reducing Fe(II) oxidizers. Autotrophic nitrate-reducing Fe(II) oxidizers have the ability to fix carbon dioxide $\left(\mathrm{CO}_{2}\right)$ and to oxidize $\mathrm{Fe}(\mathrm{II})$ coupled with complete or incomplete denitrification and can, thus, influence the fate of the groundwater pollutant nitrate and the greenhouse gases nitrous oxide $\left(\mathrm{N}_{2} \mathrm{O}\right)$ and carbon dioxide $\left(\mathrm{CO}_{2}\right)$. However, detailed knowledge about the enzymatic mechanisms of autotrophic $\mathrm{Fe}$ (II) oxidation coupled to nitrate reduction (NRFO) in the environment is lacking.

Based on several laboratory studies on autotrophic and mixotrophic nitrate-reducing Fe(II)-oxidizing bacteria [ZoBell and Upham, 1944; Rabus and Widdel, 1995; Straub et al., 1996; Buchholz-Cleven et al., 1997; Edwards et al., 2003; Beller et al., 2006; Kumaraswamy et al., 2006; Weber et al., 2006; Su et al., 2015; Zhang et al., 2015; Zhou et al., 2016], it was proposed that true autotrophic nitratereducing Fe(II)-oxidizing cultures: (i) have no need of an additional organic carbon source, (ii) maintain Fe(II) oxidation over several $(>3)$ transfers without organic carbon addition, (iii) confirm the growth of cells with only $\mathrm{Fe}$ (II), nitrate, and $\mathrm{CO}_{2}$ provided, and (iv) show $\mathrm{CO}_{2}$ uptake by the incorporation of labelled $\mathrm{CO}_{2}$ into biomass during $\mathrm{Fe}(\mathrm{II})$ oxidation [Bryce et al., 2018]. To date, there has only been one example of a stable autotrophic nitratereducing $\mathrm{Fe}(\mathrm{II})$-oxidizing bacterial culture that fits all of the above criteria: enrichment culture KS [Straub et al., 1996; He et al., 2016; Tominski et al., 2018a, b]. Culture KS was initially described by Straub et al. [1996], originates from Bremen, Germany, and is composed of $\mathrm{FeOB}$, i.e., an unclassified Gallionellaceae sp., and denitrifiers, i.e., Rhodanobacter sp. and Bradyrhizobium sp. [He et al., 2016]. These FeOB and denitrifiers in culture KS showed that species interdependencies are used as a survival strategy under the carbon-limited but Fe(II)- and nitrate-rich culture conditions [Huang et al., 2021]. Yet, it remains unknown whether additional autotrophic nitrate-reducing $\mathrm{Fe}(\mathrm{II})$-oxidizing cultures, aside from culture KS, can be cultivated to expand the diversity of known key players of autotrophic NRFO.

Nitrate-reducing Fe(II)-oxidizing bacteria were found in various types of environments [Straub and BuchholzCleven, 1998; Laufer et al., 2016] and were studied as treatments for contaminated groundwater [Su et al., 2016; Zhang et al., 2016; Kiskira et al., 2017] and constructed wetlands (i.e., wastewater treatment systems) [Song et al., 2016]. Furthermore, microorganisms of the family Gallionellaceae were widely found in a variety of natural environments [Wang et al., 2009; McBeth et al., 2013; Emerson et al., 2016; Fabisch et al., 2016; Jewell et al., 2016;
Li et al., 2017]. In addition, several observations pointed towards an involvement of Gallionellaceae spp. in NRFO processes at different habitats [Emerson et al., 2016; Jewell et al., 2016; Bethencourt et al., 2020]. However, it remained largely unknown whether these FeOB are dependent on other microbial community members, similar to the interdependencies described for culture KS [He et al., 2016; Huang et al., 2021].

Here, we explored a novel autotrophic NRFO enrichment culture, named culture BP (Bremen Pond), that originated from freshwater sediment in Bremen, Germany, and was obtained in 2015 from a pond close to the sampling site of culture KS. We studied the physiology of culture BP by analysing its geochemistry, cell numbers, and microbial community composition (via 16S rRNA gene amplicon sequencing). In addition, we used metagenomic, metatranscriptomic, and metaproteomic (meta'omics) analysis to: (i) identify the key players of NRFO in culture BP, (ii) study their potential metabolism, particularly for $\mathrm{Fe}$ (II) oxidation, denitrification, and carbon fixation, (iii) explore possible microbial interspecies interactions in culture BP, and (iv) compare the more recent culture $\mathrm{BP}$ that might resemble environmental NRFO processes more closely to the long-term established culture KS. We furthermore performed DNA- and RNA-based 16S rRNA (gene) amplicon sequencing of six sites at the original habitat of culture BP to (v) assess the in situ diversity and relative abundance of potential key players involved in NRFO at the field site.

\section{Results}

Physiology and Microbial Community Composition of the Novel Enrichment Culture BP

To explore the diversity of cultivable autotrophic FeOB contributing to NRFO in the environment, we sampled a freshwater habitat, a pond in Bremen (Germany), which is in the vicinity of the origin of the existing NRFO culture KS (a connected ditch nearby). We established the novel culture BP, maintained under the same autotrophic growth conditions as culture KS [Tominski et al., 2018a]. Culture BP was grown under autotrophic conditions (i.e., with $10 \mathrm{mM} \mathrm{Fe}(\mathrm{II})$ as an electron donor and $4 \mathrm{mM}$ nitrate as an electron acceptor in bicarbonatebuffered medium) and under heterotrophic conditions (i.e., with $5 \mathrm{mM}$ acetate as an electron donor and $4 \mathrm{mM}$ nitrate as an electron acceptor) as alternative growth condition for three successive transfers in triplicates, respectively. Samples for meta'omics analyses were collected on 


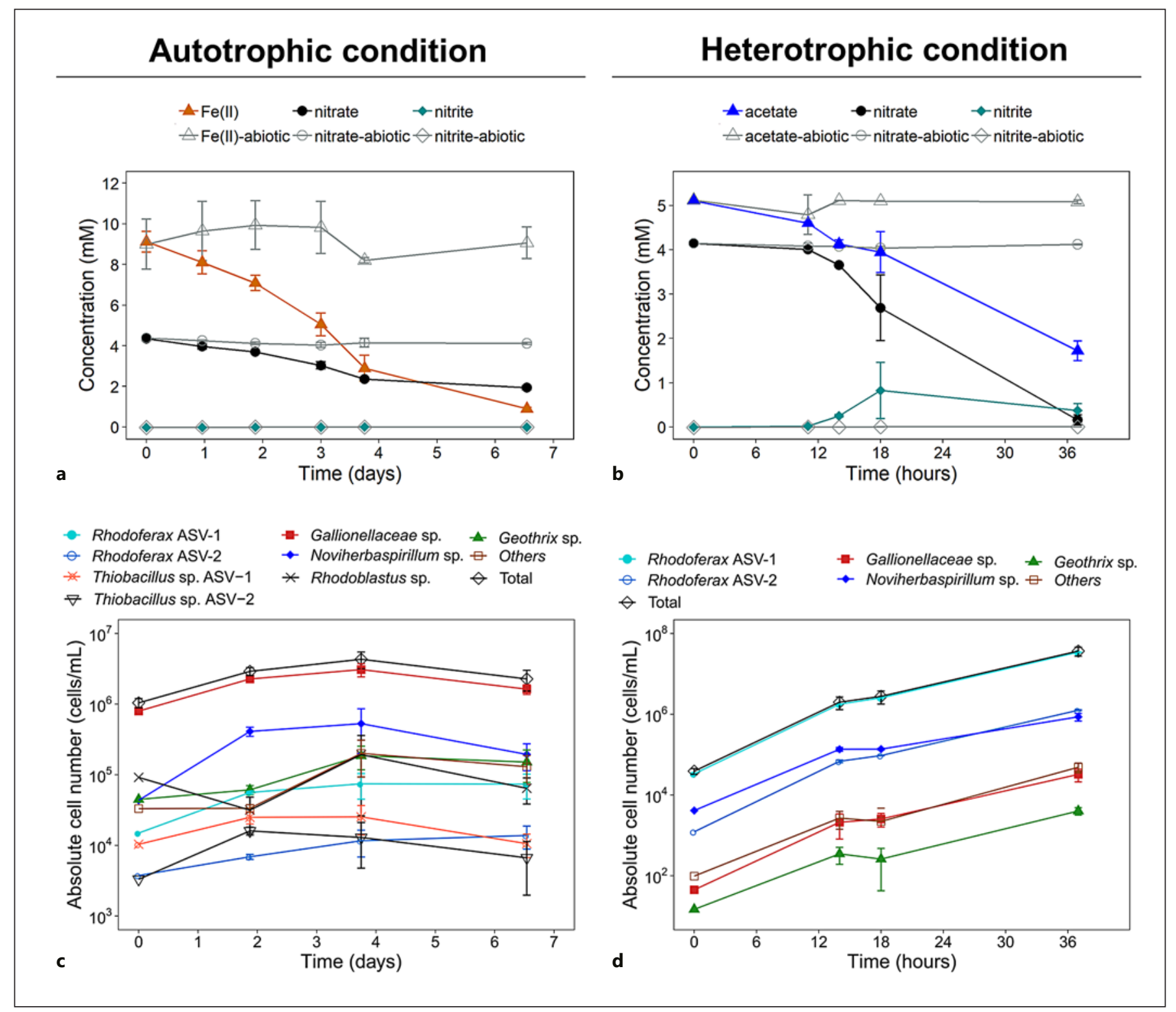

Fig. 1. Geochemistry of culture BP under autotrophic (a, c) and heterotrophic (b, d) conditions. Changes in $\mathrm{Fe}(\mathrm{II})$, nitrate and nitrite concentrations (a), acetate, nitrate and nitrite concentrations (b), and estimated absolute cell numbers (calculated using total cell

numbers and short-read 16S rRNA gene amplicon sequencing relative abundance data; (c, d), as monitored over time. Error bars represent the standard deviation of three biological replicates (except for T0).

the 2nd day under autotrophic conditions, and at $18 \mathrm{~h}$ under heterotrophic conditions; optimum sampling points were determined based on geochemical and shortread $16 \mathrm{~S}$ rRNA gene amplicon sequencing analyses conducted in parallel.

Under autotrophic conditions, $8.22 \mathrm{mM}$ of Fe(II) was oxidized in 6.5 days with an average $\mathrm{Fe}(\mathrm{II})$ oxidation rate of $1.66 \mathrm{mM} /$ day during the active growth phase (i.e., be-

tween 23 and 90 h) (Fig. 1a). This co-occurred with a reduction of $2.42 \mathrm{mM}$ of nitrate and an average nitrate reduction rate of $0.53 \mathrm{mM} /$ day, without detectable nitrite production (Fig. 1a). The average Fe(II) oxidized $_{\text {nitrate }}$ reduced stoichiometric ratio was 3.4, and cell numbers increased from $1.04 \times 10^{6}$ cells $/ \mathrm{mL}$ to $4.31 \times 10^{6}$ cells $/ \mathrm{mL}$ within 3.75 days (Fig. 1c). Under heterotrophic conditions, $3.4 \mathrm{mM}$ of acetate was consumed with an average 
acetate oxidation rate of $2.7 \mathrm{mM}$ /day during the exponential growth phase (i.e., between 11 and $37 \mathrm{~h}$ ). This co-occurred with a reduction of $4.0 \mathrm{mM}$ nitrate and an average nitrate reduction rate of $3.5 \mathrm{mM} /$ day. Nitrite increased from 0.02 to $0.8 \mathrm{mM}$ from 11 to $18 \mathrm{~h}$, but decreased again afterwards (Fig. 1b). The average ratio for acetate oxidized $_{1}$ nitrate $_{\text {reduced }}$ was 0.85 , which is higher than the theoretical stoichiometry value of 0.625 , suggesting that acetate was oxidized not only for energy generation, but also used for biomass production and nitrate was eventually reduced completely to $\mathrm{N}_{2}$. Throughout the experiment, cell numbers increased from $3.89 \times 10^{4}$ cells/mL to $3.68 \times 10^{7}$ cells/ $\mathrm{mL}$ within $37 \mathrm{~h}$ (Fig. 1d).

Under autotrophic conditions, an amplicon sequencing variant (ASV) classified as Gallionellaceae sp. dominated with $71-78 \%$ relative abundance on days $1,2,4$, and 7 (corresponding to approx. up to $3.1 \times 10^{6}$ cells $/ \mathrm{mL}$; Fig. 1c). In addition to short-reads, long-read $16 \mathrm{~S}$ rRNA gene amplicon sequencing was performed to obtain improved taxonomic identification of the microbial populations in culture BP (long-read and short-read 16S rRNA gene sequences are presented in the online suppl. data S1 and S2, respectively; for all online suppl. material, see www.karger.com/doi/10.1159/000517083). Based on long-reads, the novel unclassified Gallionellaceae sp. in culture BP shared $97.88 \%$ sequence identity with the unclassified Gallionellaceae sp. in culture KS and $96.17 \%$ with Ferrigenium kumadai (online suppl. Table S1). Other abundant ASVs were classified as Noviherbaspirillum (9-15\%; corresponding to approx. up to $5.3 \times 10^{5}$ cells $\left./ \mathrm{mL}\right)$, Geothrix (2-7\%; approx. up to $1.9 \times$ $10^{5}$ cells $/ \mathrm{mL}$ ), Rhodoferax (ASV-1 with 2-3\%; approx. up to $7.5 \times 10^{4}$ cells $/ \mathrm{mL}$ ), Thiobacillus (two ASVs with 0.4-1.6\%; approx. up to $4.1 \times 10^{4}$ cells $/ \mathrm{mL}$ ), and Rhodoblastus (1-5\%; approx. up to $1.9 \times 10^{5}$ cells/mL; Fig. $1 \mathrm{c}$ ). Under heterotrophic growth conditions, a Rhodoferax sp. (ASV-1) dominated with $84-94 \%$ relative abundance (corresponding to approx. up to $3.5 \times 10^{7}$ cells $/ \mathrm{mL}$ ) throughout the 3 rd transfer under heterotrophic conditions, while lower relative abundances were detected for ASVs classified as Noviherbaspirillum (2-11\%; approx. up to $8.6 \times 10^{5}$ cells $/ \mathrm{mL}$ ), Rhodoferax (ASV-2, only $1 \mathrm{nu}$ cleotide difference to ASV-1; 2-3\%; approx. up to $1.3 \times$ $10^{6}$ cells $/ \mathrm{mL}$ ), Gallionellaceae (up to $0.1 \%$; approx. up to $3.2 \times 10^{4}$ cells $/ \mathrm{mL}$ ), and Geothrix (up to $0.03 \%$; approx. up to $4.0 \times 10^{3}$ cells $/ \mathrm{mL}$; Fig. $1 \mathrm{~d}$ ). The long-read $16 \mathrm{~S}$ rRNA gene amplicon sequence of Rhodoferax sp. was closely related to the Curvibacter delicatus strain NBRC 14919 (98.56\% sequence identity; online suppl. Table S1).

Microbial Network in a Novel NRFO Enrichment Culture
We identified highly similar 16S rRNA gene sequences of the Rhodoblastus sp. using short- and long-read 16S rRNA amplicon sequencing compared to the metagenome-assembled genome (MAG; see below). However, for the Gallionellaceae sp., Noviherbaspirillum sp., Rhodoferax sp., Thiobacillus sp., Geothrix sp. and Betaproteobacteria bacterium, only short- and long-read sequencing offered 16S rRNA gene sequences of the respective taxa. The phylogenetic relationships of the $16 \mathrm{~S}$ rRNA gene sequences of the amplicon sequencing approach and the MAG is shown in the online supplementary Figure S1.

\section{General Characteristics of the MAGs in Culture BP}

In order to study the genomic potential of key players involved in NRFO and to use this information as reference for our meta'omics approach, twelve MAGs were retrieved from the metagenome of culture BP (online suppl. data S3). Among these twelve MAGs, six were high quality with completeness $>90 \%$ (Gallionellaceae sp., Noviherbaspirillum sp., Rhodoferax sp., Rhodoblastus sp., Thiobacillus sp. and Betaproteobacteria bacterium; online suppl. Table S1.), two had medium quality with $88 \%$ and $64.8 \%$ completeness (Ramlibacter sp. and Geothrix sp., respectively; online suppl. Table S1), and four were low quality with $<50 \%$ completeness (Geothrix sp., Mesorhizobium sp. and two unclassified MAGs), according to the definition of Bowers et al. [2017]. The four low-quality MAGs and one medium-quality MAG (Geothrix sp.) did not contain $\mathrm{Fe}(\mathrm{II})$ oxidation genes and showed only partial genes for denitrification. Thus, these five MAGs were not explored further. Additionally, the classification of the Betaproteobacteria bacterium MAG was vague, and it did not express any denitrification genes in culture BP. Therefore, we mainly focused on the remaining six MAGs, i.e., the Gallionellaceae sp., Noviherbaspirillum sp., Rhodoferax sp., Rhodoblastus sp., Thiobacillus sp. and Ramlibacter sp., representing up to $98 \%$ of the microbial community during the $\mathrm{Fe}(\mathrm{II})$ oxidation phase. These MAGs harboured genes associated with key biogeochemical cycles, including carbon, nitrogen, oxygen, and iron, in particular $\mathrm{Fe}$ (II) oxidation (Fig. 2). In order to add more evidence to gene-level information, we conducted metatranscriptomic and metaproteomic analyses to gain additional evidence at RNA and protein levels. The detection of a protein confirmed its presence and therefore suggested that the catalysed reaction of this particular protein was likely happening. Statistical meta'omics data are presented in the online supplementary Table S2 and full detected transcript and protein data are shown in the online supplementary data S4. 


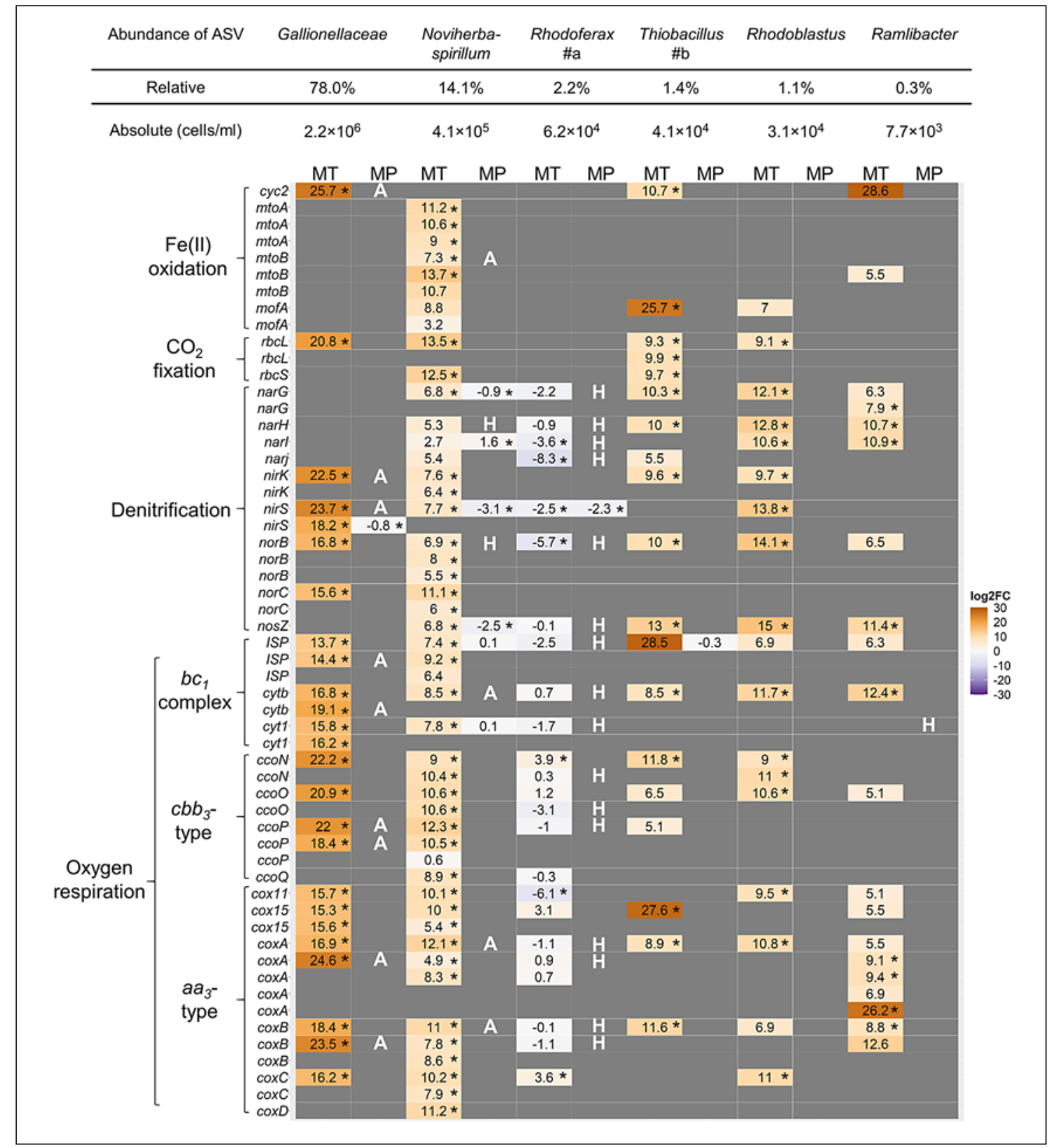

Fig. 2. The relative and estimated absolute abundance (calculated using total cell numbers and short-read 16S rRNA gene amplicon sequencing relative abundance data) of related ASVs under autotrophic conditions accompanied by fold changes $(\log 2)$ of key transcripts and proteins involved in $\mathrm{Fe}(\mathrm{II})$ oxidation, $\mathrm{CO}_{2}$ fixation, denitrification, and potential oxygen respiration genes under autotrophic conditions compared to heterotrophic conditions. Genes with several copy numbers are listed multiple times. MT, metatranscriptomic analysis; MP, metaproteomic analysis. Stars indicate significant changes (adjusted $p \leq 0.05$ ). A white " $A$ " indicates the protein was only detected under autotrophic conditions. A white " $\mathrm{H}$ " indicates the protein was only detected under heterotrophic conditions. "\#a" indicates two short-read Rhodoferax ASVs were combined, which had 100 and 99.6\% sequence identity, respectively, to the long-read Rhodoferax sp. (only one nucleotide difference). "\#b" indicates two short-read Thiobacillus ASVs were combined, which had a difference of three nucleotides to each other. 


\section{$\mathrm{Fe}$ (II) Oxidation}

To explore the pathways of $\mathrm{Fe}(\mathrm{II})$ oxidation in culture $\mathrm{BP}$, we searched for homologues of known and putative $\mathrm{Fe}(\mathrm{II})$ oxidation genes in the six MAGs, such as $c y c 2$, $m t o A$, and mofA [Corstjens et al., 1997; Castelle et al., 2008; El Gheriany et al., 2009; Liu et al., 2012; He et al., 2017]. Specifically, the $c y c 2$ gene was identified in the MAGs of the Gallionellaceae sp., Rhodoblastus sp., Thiobacillus sp., and Ramlibacter sp. At the transcript level, cyc2 was detected for the Gallionellaceae sp. and Thiobacillus sp. with significantly higher abundance (i.e., amount of detected transcript) under autotrophic conditions compared to heterotrophic conditions. In addition, the Cyc2 protein from the Gallionellaceae sp. was detected under autotrophic conditions (Fig. 2, 3). To uncover the evolutionary relationship of Cyc2 between culture $\mathrm{BP}$ and other studied organisms, we performed phylogenetic analysis of Cyc2 amino acid sequences, including those of the Gallionellaceae sp., Rhodoblastus sp., and Ramlibacter sp. (online suppl. Fig. S2). The Cyc2 of the Gallionellaceae sp. in culture BP and of Sideroxydans lithotrophicus ES-1 were closely related to each other. Overall, there appeared to be distinct clusters within the Cyc2 tree, which consisted of Gallionellaceae spp. and Zetaproteobacteria spp. (related to Mariprofundus spp.), respectively (online suppl. Fig. S2). In addition, $m t o A B$ genes were identified for the Noviherbaspirillum sp. MAG and the transcripts were at a significantly higher abundance (Fig. 2). Phylogenetic analysis of MtoAB from Noviherbaspirillum sp. demonstrated that they are closely related to the Mto $A B$ from other proposed microaerophilic FeOB (online suppl. Fig. S3) [Liu et al., 2012]. Particularly for MtoB, there appeared to a be a distinct cluster for Noviherbaspirillum (online suppl. Fig. S3). Furthermore, mofA genes were identified in the Thiobacillus sp. MAG and the transcripts were also of significantly higher abundance under autotrophic compared to heterotrophic conditions (Fig. 2).

\section{Denitrification (nirK/S, norBC)}

To investigate the mechanisms of denitrification in culture BP, we identified genes encoding the nitrate reductase (narGHI), nitrite reductase (nirK or nirS), nitric oxide reductase (nor $B C$ ), and nitrous oxide reductase (nosZ) in the MAGs of Noviherbaspirillum sp., Thiobacillus sp., Rhodoblastus sp., and Ramlibacter sp. Among these organisms, the transcripts of detected denitrification genes had significantly higher abundance under autotrophic conditions compared to heterotrophic conditions (Fig. 2). Although the denitrification pathway was incomplete in the Gallionellaceae sp., it appeared to contribute to nitrite reduction and nitric oxide reduction. Both nitrite reductase (nirK and nirS) and nitric oxide reductase (norB and nor C) transcripts from the Gallionellaceae sp. were detected at significantly higher abundance under autotrophic conditions compared to heterotrophic conditions, and the proteins of NirK/S were detected under autotrophic conditions (Fig. 2). Since the transcripts and proteins of these nirK/S genes in the MAGs of the dominant Gallionellaceae sp. were detected under autotrophic conditions (Fig. 2), this suggests that the reduction of nitrite coupled $\mathrm{Fe}$ (II) oxidation in culture BP might have occurred enzymatically. However, an abiotic contribution to the reduction of nitrite and nitric oxide by $\mathrm{Fe}(\mathrm{II})$ cannot be ruled out. Alternatively, it is possible that the Gallionellaceae sp. was expressing nitrite reductase genes to detoxify the products derived from nitrate reduction performed by other community members.

\section{Carbon Fixation}

Carbon fixation is essential for the growth of autotrophic microorganisms; thus, we explored carbon fixation pathways in culture BP under autotrophic conditions. All six MAGs contained genes involved in carbon metabolism pathways, i.e., the reductive pentose phosphate (CBB) cycle, glycolysis, tricarboxylic acid (TCA) cycle, and pentose phosphate pathway (PPP; online suppl. Table S3). More specifically, genes responsible for carbon fixation $(r b c L / S)$, encoding ribulose-1,5-bisphosphate carboxylase-oxygenase ( $\mathrm{RuBisCO}$ ), were identified in the MAGs of the Gallionellaceae sp., Noviherbaspirillum sp., Rhodoblastus sp., Thiobacillus sp., and Ramlibacter sp. (Fig. 2; online suppl. Fig. S4). The transcripts of $r b c L$ were detected at a significantly higher level under autotrophic conditions compared to heterotrophic conditions for
Fig. 3. Overview of the meta'omics results for the extracellular electron transfer system, denitrification, carbon metabolism, and oxidative phosphorylation in the three proposed key players for Fe(II) oxidation: the Gallionellaceae-BP sp. (a), Noviherbaspirillum sp. (b), and Thiobacillus sp. (c) under autotrophic conditions. Black font and lines indicate complete pathway of transcripts detected under autotrophic conditions. Grey font and lines indicate incomplete pathway of transcripts detected under autotrophic conditions. Intense blue colour shows all proteins of the complete pathway detected under autotrophic conditions. Lighter blue colour shows incomplete pathway of proteins detected under autotrophic conditions.
(For figure see next page.) 


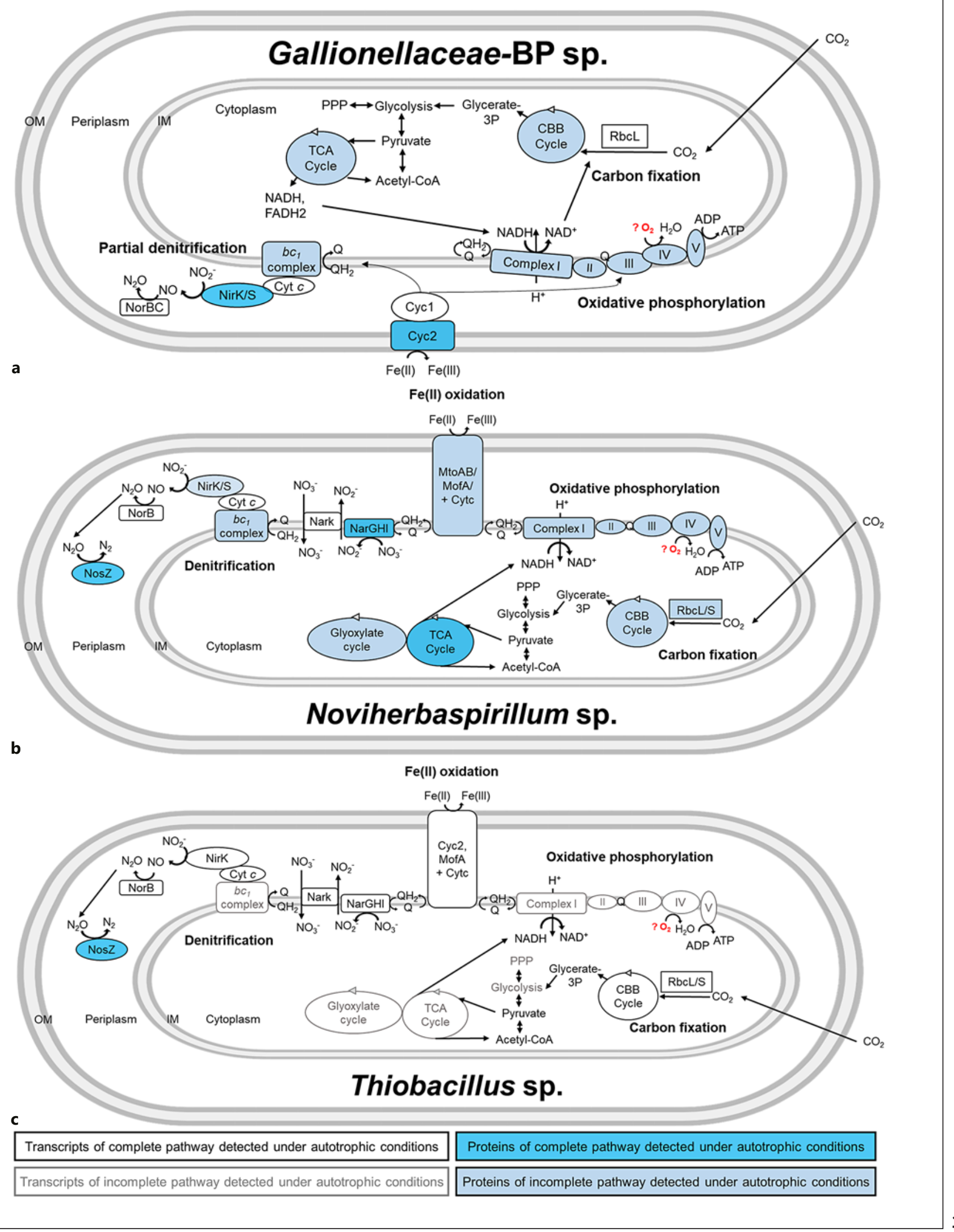


Gallionellaceae sp., Noviherbaspirillum sp., Thiobacillus sp., and Rhodoblastus sp., as well as rbcS for the Noviherbaspirillum sp. and Thiobacillus sp. (Fig. 2). However, none of the RuBisCO-related proteins were detected (Fig. 2). Phylogenetic analysis of the large-chain RuBis$\mathrm{CO}$ (RbcL; online suppl. Fig. S4A) and small-chain RuBis$\mathrm{CO}$ (RbcS; online suppl. Fig. S4B) revealed that the RbcL of the Gallionellaceae spp. from culture BP and culture KS were closely related to each other as well as to other $\mathrm{FeOB}$ (online suppl. Fig. S4A). This indicates that the RbcL of the Gallionellaceae sp. in culture BP might have the same function as the RbcL of the Gallionellaceae sp. in culture KS (online suppl. Fig. S4A).

\section{Amino Acid Biosynthesis Pathways}

Essential amino acids are vital for the survival of individual isolated organisms and, therefore, the biosynthesis of the essential amino acids of the bacterial community members in culture BP was analysed. Except for the Geothrix sp., all community members had most, if not all, essential amino acid biosynthesis pathways at least partially encoded. However, the gene encoding histidine isomerase (his A) was detected at medium confidence for the MAG of the Gallionellaceae sp. and Rhodoferax sp. with the GapMind tool [Price et al., 2020]. The genes encoding ribose-phosphate diphosphokinase ( $p r s)$ for histidine biosynthesis and homoserine kinase $(t h r B)$ for threonine biosynthesis were missing in the MAG of the Thiobacillus sp. Furthermore, the gene encoding glutamine synthetase $(g \ln A)$ for glutamine biosynthesis was missing in the MAG of the Rhodoblastus sp. In addition, the genes encoding homoserine dehydrogenase (hom) and vitamin $\mathrm{B}_{12}$-dependent methionine synthase $(\operatorname{met} H)$ for methionine biosynthesis were missing in the MAG of the Ramlibacter sp. Almost all of the essential amino acid biosynthesis pathways in the MAGs of both Gallionellaceae sp. and Noviherbaspirillum sp. were expressed (i.e., the transcripts were detected).

\section{Energy (ATP) Generation and Electron Transport Phosphorylation}

In order to identify energy generation pathways in culture $\mathrm{BP}$, we explored the different levels of gene expression and proteins for terminal electron acceptors. The genes involved in oxidative phosphorylation complexes $\mathrm{I}-\mathrm{V}$, were found in the MAGs of the Gallionellaceae sp., Noviherbaspirillum sp., Rhodoferax sp., Thiobacillus sp., Rhodoblastus sp., and Ramlibacter sp. Under autotrophic conditions, the Gallionellaceae sp., Noviherbaspirillum sp., and Rhodoblastus sp. had significantly higher expres-

Microbial Network in a Novel NRFO

Enrichment Culture sion of these respiration genes in comparison to heterotrophic conditions (Fig. 2). On the protein level, partial oxidative phosphorylation proteins were detected under autotrophic conditions for the Gallionellaceae sp. and Noviherbaspirillum sp. (Fig. 2, 3). In addition, $c b b_{3}$-type and $a a_{3}$-type cytochrome $c$ oxidase-related transcripts and proteins of complex IV, which typically have high and low affinities to oxygen, were detected under both conditions (Fig. 2), even though culture BP was grown under anoxic conditions.

\section{In situ Microbial Community Analysis}

In order to identify the in situ distribution and relative abundance of the enriched populations in culture BP, field studies were conducted in 2017. The microbial community was investigated at the sampling site of the origin of culture BP (and culture KS). To this end, sediment cores of a pond and a ditch were obtained, and DNA- and RNA-based short-read 16S rRNA (gene) sequencing was performed from a total of 50 samples. At the sampling sites, 37 different ASVs were classified as Gallionellaceae (20 ASVs were detected at the pond and 23 ASVs at the ditch; online suppl. Fig. S6); of these, eight were identified as Gallionella spp., eleven as Sideroxydans spp., three as Candidatus Nitrotoga spp. and fifteen as unclassified spp. (online suppl. Fig. S5). In the environmental samples, among these ASVs assigned to the family Gallionellaceae (accounting for up to 3.13\% relative abundance for the whole family; online suppl. Fig. S6), nine DNA-based and two RNA-based samples, corresponding to three sediment cores, revealed the same ASV (100\% sequence identity) as the dominant Gallionellaceae sp. ASV in culture BP. It accounted for up to $0.13 \%$ relative abundance and was found both at the pond and at the ditch (online suppl. Fig. S6). Therefore, the Gallionellaceae sp. in culture $\mathrm{BP}$ (i.e., the investigated sequence) was detectable, relatively widespread, and likely active at the sampling sites. The 37 different Gallionellaceae ASVs were furthermore quite diverse and affiliated, based on phylogenetic analysis, for instance with Sideroxydans lithotrophicus ES-1, Gallionella capsiferriformans ES-2, and Ferrigenium kumadai (online suppl. Fig. S5). The other members in culture BP, i.e., the Rhodoferax sp., Thiobacillus sp., and Rhodoblastus sp., were also found at the pond and the ditch with up to $0.13 \%$ relative abundance for identical ASVs as in culture BP, respectively, and up to $2.0 \%$ relative abundance for the whole genus, i.e., Rhodoblastus (online suppl. Fig. S6). Neither the Noviherbaspirillum sp. and Ramlibacter sp. in culture BP, nor the Gallionellaceae sp. in culture KS, were found within these sediment samples. 
However, our sampling procedure might just have missed them, in terms of the methodological detection limit, sampling location, or season.

\section{Discussion}

\section{A Novel Autotrophic Nitrate-Reducing Fe(II)-}

Oxidizing Enrichment Culture

Microbial NRFO is considered to be an important pathway for nitrate removal in organic-poor, Fe-abundant environments, whereby microorganisms gain electrons from $\mathrm{Fe}$ (II) for carbon fixation and for energy generation (by nitrate reduction) which is utilized for growth, ultimately transforming $\mathrm{CO}_{2}$ into biomass [Weber et al., 2006]. The enrichment of two autotrophic NRFO cultures (i.e., the culture KS and in this study culture BP) from two close-by organic-rich environments suggests that this process might be more widespread than expected and might therefore be occurring in diverse habitats.

The physiology in growing cultures of culture BP was slightly different from the results for culture KS described in previous studies. The ratio of $\mathrm{Fe}(\mathrm{II})_{\text {oxidized }} /$ nitrate $_{\text {reduced }}$ was reported in the range of 4.3-4.8 in culture KS [Straub et al., 1996; Tominski, 2016; Tominski et al., 2018a], which was higher than the $\mathrm{Fe}(\mathrm{II})_{\text {oxidized }} /$ nitrate $_{\text {reduced }}$ ratio of 3.4 in this study. Theoretically, the stoichiometry suggests that the $\mathrm{Fe}(\mathrm{II})_{\text {oxidized }} /$ nitrate $_{\text {reduced }}$ ratio should be around 5 or even higher than 5 , when the electrons required from $\mathrm{Fe}$ (II) for carbon fixation are also considered, potentially indicating incomplete denitrification or an additional electron donor (i.e., traces of dissolved organic carbon, DOC, in the growth medium) [Huang et al., 2021]. However, cell numbers increased from $1.04 \times 10^{6}$ cells $/ \mathrm{mL}$ to $4.31 \times 10^{6}$ cells $/ \mathrm{mL}$ within 4 days, and therefore the electrons must not only have derived from the DOC but also from $\mathrm{Fe}(\mathrm{II})$ oxidation.

In this study, we show that culture BP fulfils the following three criteria of autotrophic NRFO [Bryce et al., 2018]: (i) there is no need for an additional organic carbon substrate for continuous growth, (ii) the culture is able to maintain $\mathrm{Fe}(\mathrm{II})$ oxidation over several transfers without organic carbon addition, (iii) there is confirmed growth of cells with only $\mathrm{Fe}(\mathrm{II})$, nitrate, and $\mathrm{CO}_{2}$ provided. Additionally, our meta'omics data demonstrated that culture BP not only has the genetic potential but also expresses carbon fixation genes. Especially the $r b c L$ gene of the Gallionellaceae sp. in culture BP (hereafter Gallionellaceae-BP sp.) was closely related to the $r b c L$ gene of the Gallionellaceae sp. in culture KS (hereafter Gallionellace- $a e$-KS sp.), which was proven to fix ${ }^{13} \mathrm{C}$-labelled inorganic carbon [Tominski et al., 2018b]. Culture BP is therefore a novel autotrophic nitrate-reducing $\mathrm{Fe}(\mathrm{II})$-oxidizing enrichment culture that provides the opportunity to study NRFO under more environmentally relevant conditions, i.e., in a more complex microbial network with probably less laboratory evolutionary influence, compared to the long-term established culture KS.

\section{Role of Gallionellaceae for Fe(II) Oxidation in Culture $B P$ and beyond}

In this study, the dominant Gallionellaceae-BP sp. in culture BP (51-87\% relative abundance) was identified as $\mathrm{Fe}(\mathrm{II})$ oxidizer and was shown to be closely related to known FeOB, such as the unclassified Gallionellaceae-KS sp. from culture KS, a nitrate-reducing $\mathrm{Fe}(\mathrm{II})$-oxidizing culture enriched from the same freshwater habitat as culture BP [Straub et al., 1996]. Comparing meta'omics analysis of culture KS from previous studies [He et al., 2016; Huang et al., 2021] with those of culture BP in this study, some interesting differences were observed for the Gallionellaceae spp. Although both the Gallionellaceae-KS sp. and Gallionellaceae-BP sp., likely perform Fe(II) oxidation, partial denitrification, and carbon fixation, the genes encoding these functions differed. For example, the proposed $\mathrm{Fe}$ (II) oxidation genes, $c y c 2$, mto $A B$, and mof $A B$ were identified in the Gallionellaceae-KS sp. [He et al., 2016; Huang et al., 2021]. In addition, the transcripts of $c y c 2, m t o A B$ and $m o f A B$ were all detected, and the MofA protein of the Gallionellaceae-KS sp. was shown to be present [Huang et al., 2021]. For the Gallionellaceae-BP sp. $c y c 2$ was detected at DNA, RNA, and protein levels in this study. In a previous study [Castelle et al., 2008], the outer membrane-bound cytochrome $c$ Cyc2 of acidophilic $\mathrm{FeOB}$, Acidithiobacillus ferrooxidans, was purified and shown to be responsible for performing $\mathrm{Fe}$ (II) oxidation. Therefore, combined with the fact that the Gallionellace$a e$-BP sp. dominates culture $\mathrm{BP}$, these results indicate that the Cyc2 of the Gallionellaceae-BP sp. likely plays the main role for $\mathrm{Fe}(\mathrm{II})$ oxidation in culture BP under autotrophic conditions. As for denitrification, the Gallionellaceae-BP sp. harboured the genes encoding nitrite reductase (nirK/S) and nitric oxide reductase (norBC), whereas the Gallionellaceae-KS sp. harboured the genes encoding nitrate reductase (narGHI) and nitrite reductase (nirK/S). In contrast to the Gallionellaceae-KS sp., the Gallionellaceae-BP sp. possesses an NO reductase and can reduce $\mathrm{NO}$ to $\mathrm{N}_{2} \mathrm{O}$. However, both Gallionellaceae spp. in culture $\mathrm{BP}$ and culture KS require other flanking community members to complete denitrification (i.e., they are miss- 
ing the nos $Z$ gene). To investigate whether nitrite or $\mathrm{NO}$ can serve as electron acceptors and generate energy in the Gallionellaceae-BP sp., further experiments are required. Moreover, we detected cox $A B C$ encoding for $a a_{3}$-type cytochrome $c$ oxidases and belonging to the Gallionellace$a e$-BP sp. and additional community members in culture $\mathrm{BP}$, both at the transcript level and some at the protein level. Interestingly, $\operatorname{cox} A B C$ are normally observed under oxic conditions [Krab and Slater, 1979]. In contrast, in our cultivation system under autotrophic and heterotrophic conditions, we used $\mathrm{N}_{2} / \mathrm{CO}_{2}$ in the headspace and amended with $\mathrm{FeCl}_{2}$ prior to inoculation, which would have consumed any atmosphere $\mathrm{O}_{2}$ contamination during the preparation procedure. However, one possible scenario might be that the cox $A B C$ genes in the organisms in culture BP do not require oxygen to be expressed. Alternatively, there might be a so far undetected internal oxygen production pathway in at least one of the organisms in culture BP, similar to the proposed aerobic methane oxidation under anoxic conditions [Ettwig et al., 2010]. The latter hypothesis was already discussed for culture KS by He et al. [2016]. Nevertheless, these possible scenarios remain highly speculative at this point and require further investigation.

Based on 16S rRNA gene sequences, the Gallionellaceae-BP sp. was closely related to Ferrigenium kumadai, and Sideroxydans lithotrophicus strain ES-1. While Ferrigenium kumadai was isolated from rice paddy soils [Khalifa et al., 2018; Nakagawa et al., 2020], Sideroxydans lithotrophicus strain ES-1 was isolated from a groundwater-fed iron seep [Emerson and Moyer, 1997]. It is apparent, when combining our results with other studies, that Gallionellaceae spp. play important roles as $\mathrm{FeOB}$ within freshwater ecosystems. Furthermore, our in situ survey indicated a high diversity of Gallionellaceae spp. at the field site, suggesting that, in addition to the microbial populations in culture KS and culture BP, there might be a higher diversity of organisms contributing to NRFO in freshwater environments and other habitats than currently known from cultivation approaches.

\section{Contribution of Noviherbaspirillum sp. and}

Thiobacillus sp. to NRFO in Culture BP

The 2nd most abundant organism in culture BP under autotrophic conditions was the Noviherbaspirillum sp. (9-15\%). Based on 16S rRNA gene sequences, the top three closest related isolates were Noviherbaspirillum autotrophicum strain TSA66 [Ishii et al., 2017], Noviherbaspirillum agri K-1-15 [Chaudhary and Kim, 2017], and Noviherbaspirillum denitrificans TSA40 [Ishii et al.,
2017] (online suppl. Table S1). Among these isolates, only Noviherbaspirillum autotrophicum strain TSA66 was reported to be able to grow autotrophically by using $\mathrm{H}_{2}$ as an energy source to perform denitrification [Ishii et al., 2017]. However, a gene search on the Joint Genome Institute's Integrated Microbial Genome (JGI/IMG) database showed that the genome of Noviherbaspirillumautotrophicum strain TSA66 has genes encoding RuBisCO but does not have $\mathrm{Fe}(\mathrm{II})$ oxidation gene homologues, either $m t o A$ or $c y c 2$, which were detected in the Noviherbaspirillum sp. from culture BP. Therefore, the Noviherbaspirillum sp. from culture BP might have unique capabilities for NRFO within this genus.

Another potential FeOB in culture $\mathrm{BP}$ was the Thiobacillus sp., which accounted for only up to $1.6 \%$ relative abundance in culture BP and possessed the potential $\mathrm{Fe}(\mathrm{II})$ oxidation gene homologues $c y c 2$ and mofA. The top three closest related isolates based on $16 \mathrm{~S}$ rRNA gene sequences with an available genome sequence were Thiobacillus thioparus strain THI 111, Thiobacillus thioparus DSM 505, and Thiobacillus thioparus strain Starkey [Boden et al., 2012] (online suppl. Table S1). Additionally, Thiobacillus denitrificans ATCC 25259 was previously proposed as an autotrophic nitrate-reducing Fe(II)oxidizing organism [Straub et al., 1996; Beller et al., 2006]. Interestingly, only Thiobacillus thioparus DSM 505 has the Fe(II) oxidation gene $c y c 2$, and neither Thiobacillus denitrificans ATCC 25259 and Thiobacillus denitrificans RG nor Thiobacillus thiophilus DSM 19892 revealed potential $\mathrm{Fe}$ (II) oxidation genes. The copper resistance genes, $\operatorname{cop} A B$, i.e., the homologues of the putative Fe(II)oxidizing genes, $p c o A B$ [He et al., 2017], were detected in Thiobacillus denitrificans ATCC 25259, Thiobacillus denitrificans RG, Thiobacillus denitrificans DSM 12475, and Thiobacillus thiophilus DSM 19892 [Beller et al., 2006, 2013]. We therefore suggest that the Thiobacillus sp. in culture BP was also actively contributing to NRFO.

Interestingly, in culture KS, there seems to be no community member with a similar role to that of the Noviherbaspirillum sp. and Thiobacillus sp. in culture BP. For example, the 2nd most abundant organism in culture KS under autotrophic conditions, a Rhodanobacter sp., was the main organism cooperating with Gallionellaceae-KS, which is likely able to perform NRFO under autotrophic conditions [He et al., 2016; Huang et al., 2021]. The Rhodanobacter sp. likely performs $\mathrm{Fe}(\mathrm{II})$ oxidation and complete denitrification but does not have the ability to conduct carbon fixation [Huang et al., 2021]. Therefore, the isolation and the study of Noviherbaspirillum sp. and Thiobacillus sp. in culture BP, which likely contribute to 


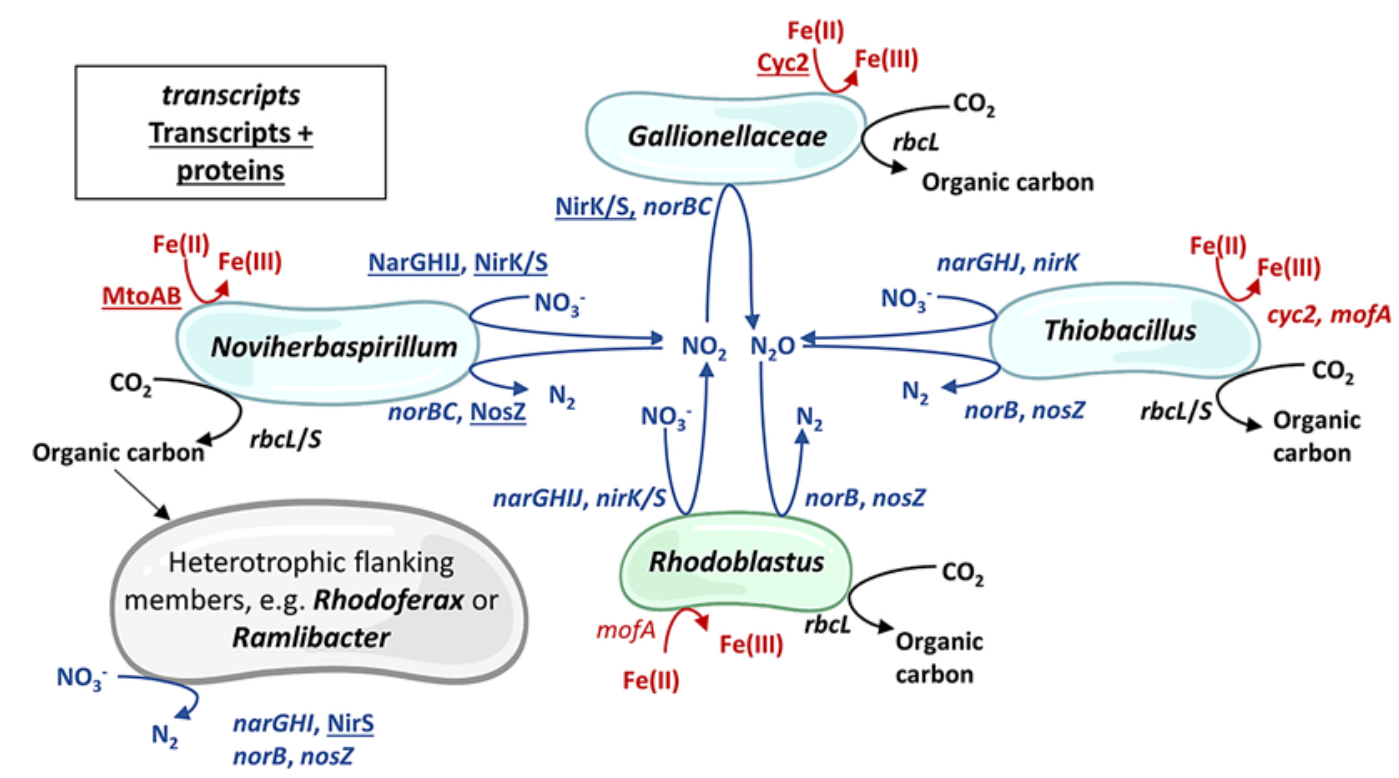

Fig. 4. Overview of the proposed microbial interactions in culture $\mathrm{BP}$, mainly essential for the organisms' survival under autotrophic conditions. The depicted putative reactions are based on the meta'omics data for Fe(II) oxidation (red arrows and font), denitrification (blue arrows and font), and $\mathrm{CO}_{2}$ fixation (black arrows and font), for the Gallionellaceae-BP sp., Noviherbaspirillum sp., Thiobacillus sp., Rhodoblastus sp., and Rhodoferax sp. The cells in

blue indicate $\mathrm{FeOB}$ with carbon fixation ability, in grey indicate heterotrophs, and in green indicate that the organism can conduct carbon fixation and potentially $\mathrm{Fe}(\mathrm{II})$ oxidation. The detected transcripts are in italic font, and a combination of both detected transcripts and proteins are with underlined font. The transcripts with significantly higher expression under autotrophic conditions compared to heterotrophic conditions are in bold font.

$\mathrm{Fe}(\mathrm{II})$ oxidation and $\mathrm{CO}_{2}$ fixation, would be beneficial in order to broaden our knowledge of autotrophic nitratereducing $\mathrm{Fe}(\mathrm{II})$-oxidizing bacteria.

\section{Expanding the Microbial Network for NRFO in}

Culture BP

Besides the Gallionellaceae-BP sp., Noviherbaspirillum sp., and Thiobacillus sp., other flanking members potentially also play a relevant role in culture BP: Rhodoblastus sp. (up to $5 \%$ relative abundance), Rhodoferax sp. (up to $3 \%$ ), and Ramlibacter sp. (up to 1\%). We identified Fe(II) oxidation gene homologues in the MAGs of the Rhodoblastus sp. (cyc2 and mofA) and the Ramlibacter sp. (cyc2 and $m t o B$ ), indicating that they can potentially play a role in $\mathrm{Fe}$ (II) oxidation, despite the fact that $c y c 2$ transcripts were not detected for the Rhodoblastus sp., and neither $c y c 2$ nor $m t o B$ transcripts for the Ramlibacter sp. were detected at significant levels. Furthermore, we observed that the genes necessary to perform full denitrification were present in these three flanking members, i.e., the Rhodoblastus sp., Rhodoferax sp., and Ramlibacter sp., and some of them were detected at significantly higher

transcript levels. Regarding carbon fixation, there was a complete $\mathrm{CBB}$ reductive pentose phosphate cycle detected in both the Rhodoblastus sp. and Ramlibacter sp., while only a partial cycle was found for the Rhodoferax sp. (online suppl. Table S3). As a result, although the relative abundance of these three flanking community members accounted for less than 5\% (respectively) throughout the autotrophic growth conditions, our findings suggest that they play partial roles in NRFO and carbon fixation (Fig. 4).

To summarize, there were five potential Fe(II)-oxidizing microbial key players in culture BP: the Gallionellaceae-BP sp., Noviherbaspirillum sp., Thiobacillus sp., Rhodoblastus sp., and Ramlibacter sp. (Fig. 4). Among these, the Gallionellaceae-BP sp., Noviherbaspirillum sp., and Thiobacillus sp. may not only perform Fe(II) oxidation but also carbon fixation (Fig. 3, 4). The resulting fixed organic carbon was likely provided to other heterotrophic community members and used for their growth (Fig. 4). During denitrification, the nitrate may be reduced by flanking community members, while the GallionellaceaeBP sp. may contribute to partial denitrification, i.e., ni- 
trite and nitric oxide reduction, and the remaining flanking community members complete the denitrification (Fig. 4). Therefore, in order to survive and indeed thrive, the dominant Gallionellaceae-BP sp. has to cooperate with other community members under autotrophic conditions (Fig. 4). In return, the heterotrophic community members may have to rely for their survival on Fe detoxification and carbon fixation likely performed by the Gallionellaceae-BP sp., Noviherbaspirillum sp., and Thiobacillus sp. (Fig. 4). In addition, our findings highlighted potential interdependencies between the microbial community members regarding amino acid biosynthesis that might be crucial for their survival as well.

Comparing the microbial network of culture BP with that of culture KS, the most active species in culture KS were the Gallionellaceae-KS sp. and the Rhodanobacter sp. under autotrophic conditions [Huang et al., 2021]. However, in culture BP, there were several active populations involved in NRFO under autotrophic conditions, including the Gallionellaceae-BP sp., Noviherbaspirillum sp., Thiobacillus sp., Rhodoblastus sp., Rhodoferax sp., and Ramlibacter sp. (Fig. 2, 3). Therefore, in contrast to culture BP, culture KS is likely characterized by a reduced microbial network, which may be due to long-term cultivation and selection of specialized organisms. The occurrence of the lower relative abundance, yet active, organisms (i.e., the Rhodoblastus sp., Rhodoferax sp., and Ramlibacter sp.) in culture BP expands the microbial network contributing to NRFO processes, which might be more similar to the complex microbial interactions in the environment.

\section{NRFO Core Features of Complex Microbial Networks including Gallionellaceae spp.}

There seems to be several core features that might be similar for autotrophic NRFO processes carried out by complex microbial networks including Gallionellaceae spp. (Fig. 4): (i) NRFO requires Fe(II) oxidation features, with several possible electron transfer pathways (i.e., different putative $\mathrm{Fe}(\mathrm{II})$-oxidizing proteins might be used); (ii) during denitrification, the Gallionellaceae spp. may contribute to partial denitrification (e.g., reducing nitrate to nitric oxide or nitrite to nitrous oxide), while the flanking microbial community members complete the denitrification; (iii) fixed organic carbon from the autotrophic lifestyle of Gallionellaceae spp. is provided to other heterotrophic community members and used for their growth; therefore, the key FeOB (i.e., the Gallionellaceae $\mathrm{sp}$.) has to cooperate with other community members under autotrophic conditions; however (iv) the identity of these flanking community members seems interchangeable. We suggest that these core features are relevant for NRFO processes in the environment as well, that NRFO is widespread, including organic-rich habitats, and that several microbial key players of NRFO in situ thrive in complex microbial networks and are yet-to-be discovered.

\section{Experimental Procedures}

Cultivation, Analytical Methods, and Cell Counts

Culture BP originated from a freshwater pond at the Max Planck Institute for Marine Microbiology, located in Bremen, Germany, sampled in 2015 (described in the online suppl. material). Since then, culture BP was transferred with $10 \%$ (v/v) inoculum, including more than 20 transfers per year from 2018, growing with $25 \mathrm{~mL}$ anoxic headspace (the $\mathrm{N}_{2} / \mathrm{CO}_{2}$ ratio was $90 / 10$ ), unfiltered, bicarbonate-buffered medium, containing $10 \mathrm{mM} \mathrm{FeCl}_{2}, 4 \mathrm{mM}$ $\mathrm{NaNO}_{3}$, vitamins, and trace elements with a final $\mathrm{pH}$ of 6.9-7.2 in $58 \mathrm{~mL}$ serum bottles [Hegler et al., 2008; Blöthe and Roden, 2009]. The incubation temperature was $28^{\circ} \mathrm{C}$ in the dark. Under heterotrophic conditions, $5 \mathrm{mM}$ acetate was used instead of $10 \mathrm{mM} \mathrm{FeCl}_{2}$ as the electron donor. Analytical methods of Fe(II), Fe(total), nitrate, nitrite and acetate concentrations were described by Tominski et al. [2018a]. Prior to cell counts, a mineral dissolution step was conducted, using the protocols obtained from personal communication with Stefanie Becker, as described in the online supplementary material. Cells were stained with BacLight Green stain (Thermo Fisher Scientific, $1 \mathrm{mM}$ stain/1 mL sample) and cell numbers were determined with an Attune NxT Flow Cytometer (Thermo Fisher Scientific) with the instrument setup adapted from the procedure of Schmidt et al. [2020], as described in the online supplementary material. Estimated absolute cell numbers were calculated by multiplying flow cytometer-based total cell numbers with short-read 16S rRNA gene amplicon sequencing relative abundance data (described below).

Experimental Setup, Biomass Sampling, DNA/RNA Co-

Extraction and Protein Extraction of Culture BP

Both autotrophic and heterotrophic conditions were used with triplicates, respectively, in the meta'omics experiment (described in the online suppl. material). Samples were taken on the 2 nd day from cultures grown under autotrophic conditions. For the control group, it was deemed essential to incubate the cultures for some time prior to the main experiment to avoid protein carryover from remaining proteins of Fe(II) oxidation. Therefore, under heterotrophic conditions, pre-cultures were first grown for two transfers sequentially with 10 and $1 \%(\mathrm{v} / \mathrm{v})$ inoculum for at least $24 \mathrm{~h}$ each. In a second step, the $3 \mathrm{rd}$ transfer with $1 \%(\mathrm{v} / \mathrm{v})$ inoculum under heterotrophic conditions was used for the experimental setup and samples were taken after approximately $18 \mathrm{~h}$. DNA, RNA, and protein samples were collected from culture BP grown under autotrophic and heterotrophic conditions. DNA/RNA co-extraction was performed according to an upscaled version of Lueders et al. [2004] (described in the online suppl. material) and used for meta'omics and short-read 16S rRNA (gene) amplicon sequencing. DNA for long-read $16 \mathrm{~S}$ rRNA gene amplicon sequencing was 
extracted using the DNEasy UltraClean Microbial Kit (QIAGEN, Hilden, Germany), according to the user manual. Protein extraction was performed according to Spät et al. [2015], as described in the online supplementary material.

\section{Sampling and DNA/RNA Co-Extraction for in situ Analysis}

For environmental sampling, six cores were obtained, three from a pond and three from a ditch near the Max Planck Institute for Marine Microbiology in Bremen. The sediment cores were separated into six different depths: $0-0.5,0.5-1,1-2,2-4,4-7$, and $7-10 \mathrm{~cm}$. DNA and RNA were co-extracted using the method published by Griffiths et al. [2000] with modifications described in the online supplementary material.

Short-Read 16S rRNA (Gene) Amplicon Sequencing

DNA from culture BP as well as DNA and cDNA (cDNA synthesis is described in the SI) from sediments were used for amplification with universal primers, i.e., 515F: GTGYCAGCMGCCGCGGTAA [Parada et al., 2016] and 806R: GGACTACNVGGGTWTCTAAT [Apprill et al., 2015] fused to Illumina adapters. The PCR, library preparation, and sequencing are detailed in the online supplementary material. Quality control, reconstruction of $16 \mathrm{~S}$ rRNA gene sequences, and taxonomic annotation was performed with nf-core/ampliseq v1.1.0 [Ewels et al., 2020; Straub et al., 2020] as outlined in the online supplementary material.

\section{Long-Read 16S rRNA Gene Amplicon Sequencing}

DNA from culture BP was used for amplification with primers 27F and 1492R. PacBio Sequel SMRT long-read amplicon sequencing was performed at the Helmholtz Zentrum München, Germany. Circular consensus sequences were analysed with DADA2 v1.10.0 [Callahan et al., 2016; Callahan et al., 2019] in R v.3.5.1 [R Development Core Team, 2018]. Further details are described in the online supplementary material.

\section{Metagenome Analysis and Draft Genome Recovery}

Library preparation and shotgun Illumina sequencing of culture BP grown under autotrophic and heterotrophic conditions were performed by CeGaT, Tuebingen, Germany. Raw read quality control, assembly, metagenome assembled genome binning and taxonomic annotation was performed with nf-core/mag v1.0.0 (https://nf-co.re/mag, DOI: 10.5281/zenodo.3589528) [Ewels et al., 2020]. Further details are described in the online supplementary material. Characteristics of the assembled metagenome can be found in the online supplementary Table S2. Twelve MAGs were obtained from the metagenome, of which eleven had $\leq 2 \%$ estimated contamination, and of these, seven had $\geq 88 \%$ estimated completeness and four had between 11 and $45 \%$ completeness. Selected MAGs are presented in the online supplementary Table S1.

The assembled metagenome and MAGs were uploaded to the Joint Genome Institute's Integrated Microbial Genome and Microbiome Expert Review (IMG/MER) pipeline (IMGAP v5.0.8) for annotation (available online at: https://img.jgi.doe.gov/cgi-bin/ mer/main.cgi) [Chen et al., 2019]. FeGenie [Garber et al., 2020] and the IMG database [Chen et al., 2019] were used to search for potential $\mathrm{Fe}(\mathrm{II})$ oxidation genes, i.e., cyc2, mto $A B$, pco $A B$, mof $A$ [He et al., 2017], for the closely related species of the community in culture $\mathrm{BP}$.

\section{Metatranscriptome Analysis}

For RNA analysis, DNAse treatment, library preparation including bacterial ribodepletion (with Illumina Stranded Total RNA Prep with Ribo-Zero Plus kit), and sequencing with $2 \times 75$ bp and 6-53 Mio clusters per sample were performed by Microsynth AG (Balgach, Switzerland) using triplicate samples of culture BP grown under autotrophic and heterotrophic conditions, respectively. For data analysis, nf-core/rnaseq v1.4.2 (https://nfco.re/rnaseq) [Ewels et al., 2020] performed quality control, mapping on the metagenome, and differential abundance testing, as detailed in the online supplementary material. The definition of a significant difference was postulated for transcripts with Benjamini and Hochberg-adjusted $p \leq 0.05$. A summary of features of the metatranscriptome is described in online supplementary Table S2.

\section{Metaproteome Analysis}

The metaproteome measurement with LC-MS/MS and MaxQuant software suite 1.6.7.0 [Cox and Mann, 2008] was conducted by the Quantitative Proteomics and Proteome Center, Tuebingen, as described in Schmitt et al. [2019] and detailed in the online supplementary material. Differential protein abundance analysis was performed with proteus v0.2.13 (https://github.com/bartongroup/ Proteus) [Gierlinski et al., 2018] (https://github.com/bartongroup/Proteus) in R v3.6.0 (https://www.R-project.org/) [R Development Core Team, 2018] (https://www.R-project.org/). Key characteristics of the metaproteomes are described in Figures 2 and 3 as well as in the online supplementary Tables S1 and S2. The definition of a significant difference was postulated for proteins with Benjamini and Hochberg-adjusted $p \leq 0.05$.

\section{Phylogenetic Analysis, Figure Illustration, and Data}

Availability

Phylogenetic analysis of Cyc2, MtoA/B, RbcL/S protein sequences of culture BP and 16S rRNA genes of Gallionellaceae spp. identified in the environmental samples were conducted with MEGA X [Kumar et al., 2018] as described in the online supplementary material. The amino acid biosynthesis pathways were determined by using the tool: GapMind: Automated Annotation of Amino Acid Biosynthesis (https://papers.genomics.lbl.gov/cgibin/gapView.cgi) [Price et al., 2020]. The physiological growth plots, cell count plots, heat map, and bubble plots (Fig. 1, 2; online suppl. Fig. S6) were constructed via R v.3.6.1 and its working interface RStudio (https://www.R-project.org/and http://www.rstudio.com/) [RStudio Team, 2019; R Development Core Team, 2020]. Figure illustrations of cell shapes (Fig. 3, 4) were adapted from "Icons $>$ Cell Membranes $>$ Simplified Bilayer Membranes" and "Icons > Cell Structures > Organelles," respectively, by using BioRender.com (2021; https://app.biorender.com/biorender-templates). The datasets presented in this study can be found in online repositories. The names of the repositories and accession numbers are described below and in the online supplementary Table S4. Raw sequencing data and metagenome assembly were deposited at the Sequence Reads Archive (SRA; https://www.ncbi.nlm.nih.gov/ bioproject/PRJNA693457). The mass spectrometry proteomics data have been deposited to the ProteomeXchange Consortium via the PRIDE [Perez-Riverol et al., 2019] partner repository with the dataset identifier PXD023710. The IMG metagenome ID for BP culture is 3300036710 and the corresponding accession numbers of MAGs are: Gallionellaceae sp. 2831290873; Noviherbaspirillum sp. 2831285802; Rhodoferax sp. 2840079448; Thiobacillus sp. 
2840071692; Rhodoblastus sp. 2840074988; Ramlibacter sp. 2840082686; Geothrix sp. 2840087968, and Betaproteobacteria bacterium 2840090052 (online suppl. Table S1and S2) [Chen et al., 2019]. The other four MAGs did not reach the quality criteria for submission to IMG.

\section{Acknowledgements}

We thank Shun Li for obtaining the sediment sample for cultivation, Wiebke Ruschmeier for setting up the enrichment culture, Jens Harder, Ingrid Kunze, Timm Bayer, Lea Sauter, and Casey Bryce for assistance during sampling, Tillmann Lueders, Zhe Wang, and Natalia Jakus for PacBio library preparation and sequencing, Irina Droste-Borel for metaproteomic measurement, Verena Nikeleit, Ellen Roehm, and Franziska Schaedler for acetate, nitrate and nitrite measurement, as well as Stefanie Becker for developing the cell count methods for flow cytometry.

\section{Statement of Ethics}

As no human or animal research materials were involved in this study, the paper is exempt from ethical committee approval.

\section{Conflict of Interest Statement}

The authors have no conflicts of interest to declare.

\section{Funding Sources}

This work was supported by the German Research Foundation (Deutsche Forschungsgemeinschaft, DFG)-funded research training group RTG 1708 "Molecular Principles of Bacterial Survival Strategies." Daniel Straub was funded by the Institutional Strategy of the University of Tuebingen (DFG, ZUK63). Andreas Kappler is funded by the DFG under Germany's Excellence Strategy, cluster of Excellence EXC2124 (project ID 390838134). Nia Blackwell was funded by the Collaborative Research Center 1253 CAMPOS (Project 5: Fractured Aquifers) from the DFG (grant No. SFB 1253/1 2017). Sara Kleindienst is funded by an Emmy-Noether fellowship from the DFG (grant No. 326028733).

\section{Author Contributions}

Y.-M.H.: experimental design and conduct, metagenomic, metaproteomic, metatranscriptomic and 16S rRNA amplicon sequencing data interpretation, manuscript writing and revising, final approval of the version to be published. D.S.: experimental design, metagenomic, metaproteomic, metatranscriptomic and $16 \mathrm{~S}$ rRNA amplicon sequencing bioinformatics analysis, manuscript writing and revising, final approval of the version to be published. A.K.: experimental design, supervision, laboratory resources, manuscript revising, final approval of the version to be published. N.S.: metagenomic and 16S rRNA amplicon sequencing data interpretation, manuscript writing and revising, final approval of the version to be published. N.B.: experimental design, supervision, manuscript revising, final approval of the version to be published. S.K.: experimental design, supervision, funding acquisition, laboratory resources, manuscript revising, final approval of the version to be published.

\section{References}

Apprill A, McNally S, Parsons R, Weber L. Minor revision to V4 region SSU rRNA 806R gene primer greatly increases detection of SAR11 bacterioplankton. Aquat. Microb. Ecol.. 2015; 75(2):129-37.

Beller HR, Chain PS, Letain TE, Chakicherla A, Larimer FW, Richardson PM, et al. The genome sequence of the obligately chemolithoautotrophic, facultatively anaerobic bacterium Thiobacillus denitrificans. J Bacteriol. 2006 Feb;188(4):1473-88.

Beller HR, Zhou P, Legler TC, Chakicherla A, Kane S, Letain TE, et al. Genome-enabled studies of anaerobic, nitrate-dependent iron oxidation in the chemolithoautotrophic bacterium Thiobacillus denitrificans. Front Microbiol. 2013 2013-August-27;4(249):249.

Bethencourt L, Bochet O, Farasin J, Aquilina L, Borgne TL, Quaiser A, et al. Genome reconstruction reveals distinct assemblages of Gallionellaceae in surface and subsurface redox transition zones. FEMS Microbiol Ecol. 2020 May 1;96(5):fiaa036.

Blöthe M, Roden EE. Composition and activity of an autotrophic $\mathrm{Fe}(\mathrm{II})$-oxidizing, nitrate-re- ducing enrichment culture. Appl Environ Microbiol. 2009 Nov;75(21):6937-40.

Boden R, Cleland D, Green PN, Katayama Y, Uchino Y, Murrell JC, et al. Phylogenetic assessment of culture collection strains of Thiobacillus thioparus, and definitive 16S rRNA gene sequences for $\mathrm{T}$. thioparus, T. denitrificans, and Halothiobacillus neapolitanus. Arch Microbiol. 2012 Mar;194(3):187-95.

Bowers RM, Kyrpides NC, Stepanauskas R, Harmon-Smith M, Doud D, Reddy TBK, et al. Minimum information about a single amplified genome (MISAG) and a metagenomeassembled genome (MIMAG) of bacteria and archaea. Nat Biotechnol. 2017 Aug 8;35(8): 725-31.

Bryce C, Blackwell N, Schmidt C, Otte J, Huang YM, Kleindienst S, et al. Microbial anaerobic $\mathrm{Fe}(\mathrm{II})$ oxidation - Ecology, mechanisms and environmental implications. Environ Microbiol. 2018 Oct;20(10):3462-83.

Buchholz-Cleven BEE, Rattunde B, Straub KL. Screening for genetic diversity of isolates of anaerobic $\mathrm{Fe}(\mathrm{II})$-oxidizing bacteria using DGGE and whole-cell hybridization. System- atic and Applied Microbiology. 1997;20(2): 301-9.

Callahan BJ, McMurdie PJ, Rosen MJ, Han AW, Johnson AJ, Holmes SP. DADA2: high-resolution sample inference from Illumina amplicon data. Nat Methods. 2016 Jul;13(7):581-3.

Callahan BJ, Wong J, Heiner C, Oh S, Theriot CM, Gulati AS, et al. High-throughput amplicon sequencing of the full-length $16 \mathrm{~S}$ rRNA gene with single-nucleotide resolution. $\mathrm{Nu}-$ cleic Acids Res. 2019 Oct 10;47(18):e103.

Castelle C, Guiral M, Malarte G, Ledgham F, Leroy $\mathrm{G}$, Brugna $\mathrm{M}$, et al. A new iron-oxidizing/ $\mathrm{O}_{2}$-reducing supercomplex spanning both inner and outer membranes, isolated from the extreme acidophile Acidithiobacillus ferrooxidans. J Biol Chem. 2008 Sep 19;283(38): 25803-11.

Chaudhary DK, Kim J. Noviherbaspirillum agri sp. nov., isolated from reclaimed grassland soil, and reclassification of Herbaspirillum massiliense (Lagier et al., 2014) as Noviherbaspirillum massiliense comb. nov. Int J Syst Evol Microbiol. 2017 May;67(5):150815. 
Chen IA, Chu K, Palaniappan K, Pillay M, Ratner A, Huang J, et al. IMG/M v.5.0: an integrated data management and comparative analysis system for microbial genomes and microbiomes. Nucleic Acids Res. 2019 Jan 8;47(D1): D666-D77.

Corstjens PLAM, de Vrind JPM, Goosen T, de Jong EWdV. Identification and molecular analysis of the Leptothrix discophoraSS1mofAgene, a gene putatively encoding a manganese-oxidizing protein with copper domains. Geomicrobiol J. 1997;14(2):91-108.

Cox J, Mann M. MaxQuant enables high peptide identification rates, individualized p.p.b.range mass accuracies and proteome-wide protein quantification. Nat Biotechnol. 2008; 26(12):1367-72.

Edwards KJ, Rogers DR, Wirsen CO, McCollom TM. Isolation and characterization of novel psychrophilic, neutrophilic, Fe-oxidizing, chemolithoautotrophic alpha- and gammaproteobacteria from the deep sea. Appl Environ Microbiol. 2003;69(5):2906-13.

El Gheriany IA, Bocioaga D, Hay AG, Ghiorse WC, Shuler ML, Lion LW. Iron requirement for $\mathrm{Mn}$ (II) oxidation by Leptothrix discophora SS-1. Appl Environ Microbiol. 2009 Mar; 75(5):1229-35.

Emerson D, Moyer C. Isolation and characterization of novel iron-oxidizing bacteria that grow at circumneutral pH. Appl Environ Microbiol. 1997;63(12):4784-92.

Emerson JB, Thomas BC, Alvarez W, Banfield JF. Metagenomic analysis of a high carbon dioxide subsurface microbial community populated by chemolithoautotrophs and bacteria and archaea from candidate phyla. Environ Microbiol. 2016 Jun;18(6):1686-703.

Ettwig KF, Butler MK, Le Paslier D, Pelletier E, Mangenot S, Kuypers MM, et al. Nitrite-driven anaerobic methane oxidation by oxygenic bacteria. Nature. 2010;464(7288):543-8.

Ewels PA, Peltzer A, Fillinger S, Patel H, Alneberg J, Wilm A, et al. The nf-core framework for community-curated bioinformatics pipelines. Nat Biotechnol. 2020;38(3):276-8.

Fabisch M, Freyer G, Johnson CA, Büchel G, Akob DM, Neu TR, et al. Dominance of 'Gallionella capsiferriformans' and heavy metal association with Gallionella-like stalks in metal-rich pH 6 mine water discharge. Geobiology. 2016;14(1):68-90.

Garber AI, Nealson KH, Okamoto A, McAllister SM, Chan CS, Barco RA, et al. FeGenie: a comprehensive tool for the identification of iron genes and iron gene neighborhoods in genome and metagenome assemblies. Front Microbiol. 2020;11:37.

Gierlinski M, Gastaldello F, Cole C, Barton GJ. Proteus: an R package for downstream analysis of MaxQuant output. bioRxiv. 2018:416511.

Griffiths RI, Whiteley AS, O'Donnell AG, Bailey MJ. Rapid method for coextraction of DNA and RNA from natural environments for analysis of ribosomal DNA- and rRNA-based microbial community composition. Appl Environ Microbiol. 2000 Dec;66(12):5488-91.
He S, Barco RA, Emerson D, Roden EE. Comparative genomic analysis of neutrophilic iron(II) oxidizer genomes for candidate genes in extracellular electron transfer. Front Microbiol. 2017;8(1584):1584.

He S, Tominski C, Kappler A, Behrens S, Roden EE. Metagenomic analyses of the autotrophic $\mathrm{Fe}(\mathrm{II})$-oxidizing, nitrate-reducing enrichment culture KS. Appl Environ Microbiol. 2016 May;82(9):2656-68.

Hegler F, Posth NR, Jiang J, Kappler A. Physiology of phototrophic iron(II)-oxidizing bacteria: implications for modern and ancient environments. FEMS Microbiol Ecol. 2008 Nov; 66(2):250-60.

Huang Y, Straub D, Blackwell N, Kappler A, Kleindienst S. Meta-omics reveal Gallionellaceae and Rhodanobacter as interdependent key players for $\mathrm{Fe}(\mathrm{II})$ oxidation and nitrate reduction in the autotrophic enrichment culture KS. Appl Environ Microbiol. 2021. doi: 10.1128/AEM.00496-21.

Ishii S, Ashida N, Ohno H, Segawa T, Yabe S, Otsuka $S$, et al. Noviherbaspirillum denitrificans sp. nov., a denitrifying bacterium isolated from rice paddy soil and Noviherbaspirillum autotrophicum sp. nov., a denitrifying, facultatively autotrophic bacterium isolated from rice paddy soil and proposal to reclassify Herbaspirillum massiliense as Noviherbaspirillum massiliense comb. nov. Int J Syst Evol Microbiol. 2017 Jun;67(6):1841-8.

Jewell TN, Karaoz U, Brodie EL, Williams KH, Beller HR. Metatranscriptomic evidence of pervasive and diverse chemolithoautotrophy relevant to C, S, N and Fe cycling in a shallow alluvial aquifer. ISME J. 2016 2016/09/01; 10(9):2106-17.

Khalifa A, Nakasuji Y, Saka N, Honjo H, Asakawa $S$, Watanabe T. Ferrigenium kumadai gen. nov., sp. nov., a microaerophilic iron-oxidizing bacterium isolated from a paddy field soil. Int J Syst Evol Microbiol. 2018 Aug;68(8): 2587-92.

Kiskira K, Papirio S, van Hullebusch ED, Esposito G. Fe(II)-mediated autotrophic denitrification: a new bioprocess for iron bioprecipitation/biorecovery and simultaneous treatment of nitrate-containing wastewaters. Int Biodeterior Biodegradation. 2017;119:631-48.

Kumar S, Stecher G, Li M, Knyaz C, Tamura K. MEGA X: molecular evolutionary genetics analysis across computing platforms. Mol Biol Evol. 2018 Jun 1;35(6):1547-9.

Kumaraswamy R, Sjollema K, Kuenen G, van Loosdrecht M, Muyzer G. Nitrate-dependent [Fe(II)EDTA]2- oxidation by Paracoccus ferrooxidans sp. nov., isolated from a denitrifying bioreactor. Syst Appl Microbiol. 2006 Jun; 29(4):276-86.

Laufer K, Røy H, Jørgensen BB, Kappler A. Evidence for the existence of autotrophic nitratereducing $\mathrm{Fe}$ (II)-oxidizing bacteria in marine coastal sediment. Appl Environ Microbiol. 2016 Oct 15;82(20):6120-31.

Li J, Cui J, Yang Q, Cui G, Wei B, Wu Z, et al. Oxidative weathering and microbial diversity of an inactive seafloor hydrothermal sulfide chimney. Front Microbiol. 2017;8(1378): 1378.

Liu J, Wang Z, Belchik SM, Edwards MJ, Liu C, Kennedy DW, et al. Identification and characterization of MtoA: a decaheme c-type cytochrome of the neutrophilic $\mathrm{Fe}$ (II)-oxidizing bacterium Sideroxydans lithotrophicus ES-1. Front Microbiol. 2012;3(37):37.

Lueders T, Manefield M, Friedrich MW. Enhanced sensitivity of DNA- and rRNA-based stable isotope probing by fractionation and quantitative analysis of isopycnic centrifugation gradients. Environ Microbiol. 2004 Jan; 6(1):73-8.

McBeth JM, Fleming EJ, Emerson D. The transition from freshwater to marine iron-oxidizing bacterial lineages along a salinity gradient on the Sheepscot River, Maine, USA. Environ Microbiol Rep. 2013;5(3):453-63.

Nakagawa K, Murase J, Asakawa S, Watanabe T. Involvement of microaerophilic iron-oxidizing bacteria in the iron-oxidizing process at the surface layer of flooded paddy field soil. J Soils Sediments. 2020;20(11):4034-41.

Parada AE, Needham DM, Fuhrman JA. Every base matters: assessing small subunit rRNA primers for marine microbiomes with mock communities, time series and global field samples. Environ Microbiol. 2016;18(5): 1403-14.

Perez-Riverol Y, Csordas A, Bai J, Bernal-Llinares M, Hewapathirana S, Kundu DJ, et al. The PRIDE database and related tools and resources in 2019: improving support for quantification data. Nucleic Acids Res. 2019 Jan 8; 47(D1):D442-50.

Price MN, Deutschbauer AM, Arkin AP. GapMind: automated annotation of amino acid biosynthesis. mSystems. 2020;5(3):e00291-20.

R Development Core Team . A language and environment for statistical computing. Vienna: $\mathrm{R}$ Foundation for Statistical Computing; 2018.

R Development Core Team . A language and environment for statistical computing. Vienna: $\mathrm{R}$ Foundation for Statistical Computing; 2020.

Rabus R, Widdel F. Anaerobic degradation of ethylbenzene and other aromatic hydrocarbons by new denitrifying bacteria. Arch Microbiol. 1995;163(2):96-103.

RStudio Team . RStudio: integrated development for R. Boston: RStudio Inc.; 2019.

Schmidt C, Nikeleit V, Schaedler F, Leider A, Lueder U, Bryce C, et al. Metabolic responses of a phototrophic co-culture enriched from a freshwater sediment on changing substrate availability and its relevance for biogeochemical iron cycling. Geomicrobiol J. 2021:38(3): 267-81.

Schmitt M, Sinnberg T, Nalpas NC, Maass A, Schittek B, Macek B. Quantitative proteomics links the intermediate filament nestin to resistance to targeted BRAF inhibition in melanoma cells. Mol Cell Proteomics. 2019 Jun; 18(6):1096-109. 
Song X, Wang S, Wang Y, Zhao Z, Yan D. Addition of $\mathrm{Fe} 2+$ increase nitrate removal in vertical subsurface flow constructed wetlands. Ecol Eng. 2016;91:487-94.

Spät P, Maček B, Forchhammer K. Phosphoproteome of the cyanobacterium Synechocystis sp. PCC 6803 and its dynamics during nitrogen starvation. Front Microbiol. 2015;6:248.

Straub D, Blackwell N, Langarica-Fuentes A, Peltzer A, Nahnsen S, Kleindienst S. Interpretations of environmental microbial community studies are biased by the selected 16S rRNA (gene) amplicon sequencing pipeline. Front Microbiol. 2020;11:550420.

Straub KL, Benz M, Schink B, Widdel F. Anaerobic, nitrate-dependent microbial oxidation of ferrous iron. Appl Environ Microbiol. 1996; 62(4): 1458.

Straub KL, Buchholz-Cleven BE. Enumeration and detection of anaerobic ferrous iron-oxidizing, nitrate-reducing bacteria from diverse European sediments. Appl Environ Microbiol. 1998;64(12):4846-56.

Su JF, Shao S, Huang Tl, Ma F, Yang S, Zhou Z, et al. Anaerobic nitrate-dependent iron(II) oxidation by a novel autotrophic bacterium, Pseudomonas sp. SZF15. J Environ Chem Eng. 2015;3(3):2187-93.
Su JF, Shi JX, Huang TL, Ma F, Lu JS, Yang SF. Effect of nitrate concentration, $\mathrm{pH}$, and hydraulic retention time on autotrophic denitrification efficiency with $\mathrm{Fe}(\mathrm{II})$ and $\mathrm{Mn}(\mathrm{II})$ as electron donors. Water Sci Technol. 2016; 74(5):1185-92.

Tominski C. Genetic and physiological mechanisms of nitrate-dependent $\mathrm{Fe}$ (II) oxidation in the chemolithoautotrophic enrichment culture KS. Tuebingen: Eberhard Karls University of Tuebingen; 2016. p. 158.

Tominski C, Heyer H, Lösekann-Behrens T, Behrens S, Kappler A. Growth and population dynamics of the anaerobic Fe(II)-oxidizing and nitrate-reducing enrichment culture KS. Appl Environ Microbiol. 2018a May;84(9):e0217317.

Tominski C, Lösekann-Behrens T, Ruecker A, Hagemann N, Kleindienst S, Mueller CW, et al. Insights into carbon metabolism provided by fluorescence in situ hybridization-secondary ion mass spectrometry imaging of an autotrophic, nitrate-reducing, $\mathrm{Fe}(\mathrm{II})$-oxidizing enrichment culture. Appl Environ Microbiol. 2018b May 1;84(9):19.

Wang J, Muyzer G, Bodelier PL, Laanbroek HJ. Diversity of iron oxidizers in wetland soils revealed by novel $16 \mathrm{~S}$ rRNA primers targeting Gallionella-related bacteria. ISME J. 2009; 3(6):715-25.
Weber KA, Pollock J, Cole KA, O'Connor SM, Achenbach LA, Coates JD. Anaerobic nitratedependent iron(II) bio-oxidation by a novel lithoautotrophic betaproteobacterium, strain 2002. Appl Environ Microbiol. 2006 Jan; 72(1):686-94.

Zhang H, Wang H, Yang K, Sun Y, Tian J, Lv B. Nitrate removal by a novel autotrophic denitrifier (Microbacterium sp.) using $\mathrm{Fe}(\mathrm{II})$ as electron donor. Ann Microbiol. 2015 2015/06/01;65(2):1069-78.

Zhang X, Li A, Szewzyk U, Ma F. Improvement of biological nitrogen removal with nitrate-dependent $\mathrm{Fe}$ (II) oxidation bacterium Aquabacterium parvum B6 in an up-flow bioreactor for wastewater treatment. Bioresour Technol. 2016;219:624-31.

Zhou J, Wang H, Yang K, Ji B, Chen D, Zhang H, et al. Autotrophic denitrification by nitratedependent $\mathrm{Fe}(\mathrm{II})$ oxidation in a continuous up-flow biofilter. Bioprocess Biosyst Eng. 2016;39(2):277-84.

ZoBell CE, and Upham, H. C. A list of marine bacteria including descriptions of sixty new species. Berkeley: University of California Press; 1944. 